\title{
X-RAY PHOTOEMISSION STUDIES OF RARE EARTH HARD MAGNETS
}

J. R. Cuthill, A. J. McAlister, and N. E. Erickson

National Bureau of Standards, Gaithersburg, Md. 20760

R. E. Watson

Brookhaven National Laboratory, ${ }^{\dagger}$ Upton, N. Y. 11973

\section{ABSTRACT}

The present investigation attempts to probe the electronic properties of the $\mathrm{RCO}_{5}$ compounds by $\mathrm{x}$-ray photoelectron spectroscopy studies of $\mathrm{SmCO}_{5}$ and $\mathrm{PrCO}_{5}$ and endeavors to relate the results to the properties of the pure metals and to the picture developed to describe the magnetism of the compounds. The results indicate a valence band structure which is similar to that of $C_{0}$ metal; there is the suggestion that if there is charge flow it is off the rare-earth sites; there appears to be significant change in the $4 \mathrm{f}$ spectra from that of the pure rare-earth metals; and there is a suggestion of fod hybridization.

\section{INTRODUCTION}

The rare-earth cobalt compounds form an interesting and valuable class of magnetic materials as is evidenced by sessions elsewhere in this conference. It would appear ${ }^{l}$ that the magnetism is primarily associated with the Co sub-lattice, the rare-earths causing that sub-lattice to be of lower coordination than Co metal. Given the Co magnetization, the rare-earths align antiferromagnetically in its rield. In the present study, $x$-ray photoelectron spectral data is obtained on the valence bands and pertinent inner levels in pursuit of the relationship between the magnetic behavior and the electronic structure of these compounds.

\section{EXPERIMENTAL DETAILS}

The $\mathrm{SmCo}_{5}$ and $\mathrm{PrCo}_{5}$ compounds were made using high-purity alumina crucibles in an induction furnace backfilled with Tigettered. argon to a pressure of 600 torr. Reaction between the rare-earth and crucible was minimized by adding the rare-earth after melting the cobalt. The charge was held molten for two minutes after the addition of the rare-earth to insure complete reaction of the rare-earth, then poured into a chilled steel mold while still under argon. Metallographic inspection of both $\mathrm{SmCO}_{5}$ and $\mathrm{PrCO}_{5}$ ingots, in their as-cast condition, revealed approximately $10 \%$ of a second phase which was presumed to be $\mathrm{R}_{2} \mathrm{Co}_{17}$. Following Ray and 
Millott ${ }^{2}$, the $\mathrm{SmCO}_{5}$ and $\mathrm{PrCO}_{5}$ ingots were then homogenized $50 \mathrm{sr} 12$ hours at $1220^{\circ} \mathrm{C}$ and $1115^{\circ} \mathrm{C}$, respectively, which in each case is $\sim 115^{\circ} \mathrm{C}$ below the perftectic decomposition temperature of the respective $\mathrm{RCO}_{5}$ phase. Re-examination of the microstructure revealed that this homogenization treatment had resilted in a single phase throughout. X-ray diffraction studies confirmed the homogenization and showed the samples to have the $\mathrm{CaCu}_{5}$ structure.

The $x$-ray photoelectron spectra were obtained on a modified A.E.I. spectrometer, ${ }^{*}$ equipped with a P.E.I. Ion gun for cleaning. The specimens were given a metallographic polish before insertion in the spectrometer and then were elther argon-1on or neon-1on cleaned in situ. The cleaning was repeated at approximately 15-minute intervals. All spectra were recorded digitally at $0.2 \mathrm{eV}$ intervals on a multichannel analyzer. The pressure in the specimen chamber during recording was less than $5 \times 10^{-9}$ torr.

\section{RESULTS}

The core level spectra of $\mathrm{Co}$ in $\mathrm{PrCO}_{5}$ and $\mathrm{SmCO}_{5}$ are unshifted from, and are of almost identical structure and breadth, to the spectra of pure Co. As an example, the $3 \mathrm{~s}$ spectrum of Co in Sinco 5 is compared with that of Co metal ${ }^{+}$in Fig. 1. There is no evidence of different spectra for the two crystallographically nonequivalent

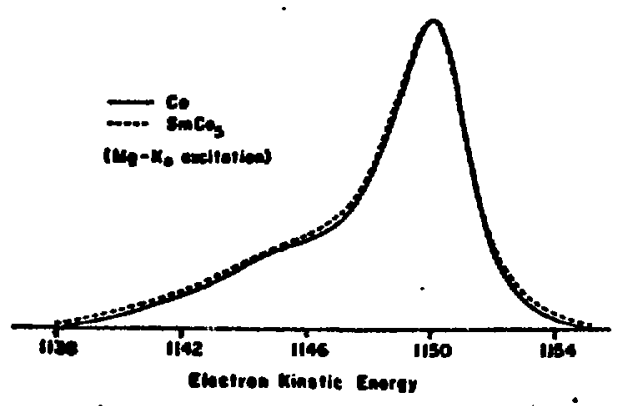

Fig. 1. The Co-3s spectrum. Co sites which occur in the compound, suggesting that the sites are electronically very similar. Either spectrum is a composite of lines associated with the coupling of the $3 s$ hole with the d shell. The two spectra are almost 1dentical, suggesting that the Co spin moment in the alloy is essentially that of Co metal, consistent with the neutron diffraction data. 3 The fact that the co spectrum does not shift on going to the alloy is not compelling evidence against charge flow between $\mathrm{Sm}$ and Co sites because any chemical shift of the core level will be accompanied by a similar chemical shift of the $d$ bands and hence of the Ferml level, $\varepsilon_{f}$, which falls high in the $d$ bands. In other words there will be a tendency of the core level to be "pinned" to $\varepsilon_{f}$. Since photoemission measures ${ }^{4}$ the

*The mention of a manufacturer by name is for identification only and does not Imply endorsement of the product by the government.

+After argon-ion cleaning, to be compatible with the treatment gien the $\mathrm{PrCO}_{5}, \mathrm{Sm}$, and $\mathrm{SmCo}_{5}$. No significant oxygen or carbon was detected. A narrower valence band spectrum has been obtained after baking out the Co. 


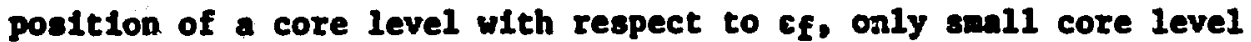
shifts are expected for high density of states transition metals such as Co. The rare-earth core levels display $0-1 / 2 \mathrm{eV}$ increases in bindIns energy, relative to $\varepsilon_{f}$, in the compounds. Th1s, taken with the trend ${ }^{5}$ in work function upon going from the rare-earthe to $\mathrm{Co}$, susgests that there may be sone charge flow off the rare-earth sites. A quantitative eatiwate of the charge flow will not be attempted here.

The valence band region of the apectrum is displayed for PrCo 5

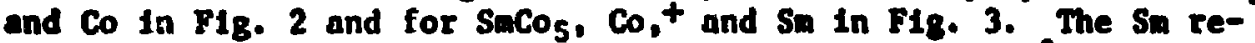
oult is essentially Identicnl to that of Baer and Busch ${ }^{6}$. The $\mathrm{SmCO}_{5}$ opectrum represents a sum of scans, some of which werc recorded after Ar-ion cleaning, and the remainder after Ne-ton cleaning. The structure 11-12 volts below $\varepsilon_{f}$ is Identifled as due to the argon because it was not present after He-ion cleaning. Granted that a Co atom has approximately eight d band electrons whereas Sm or Pr have n2 and that there are five times as many co atons, the spectrum $0-5$ eV below $c_{f} 18$ presumbly associated primarily with co $3 d$ character. The band widihs of both compounds are about the sane as that of pure co metal. One would expect that the unhybridized co site $3 d$ bands would be markedly narrower in the compound becsuse, while Co-Co distances are about the sane, the number of Co-Co near neighbors $182 / 3$ and $3 / 4$ (for the two types of Co sites) that of the pure metal. Band width 18 expected to be proportional to the number of near nelghbors in a tight binding scheme for an ordered compound. Whether rare-earth-cobalt hybridization is responsible for the lack of narrowing reanins to be seen?, but there appears to be some evidence of hybridization. Baer and Busch 6 obtained a single $4 f$ line For Pr metal which lies at an energy well Inside the compound's d bands as is indicated by the arrow in Fig. 2. No discernable peaking 1s seen in the PrCos spectrum at this energy but admixture of the $f$ level with $d$ bands, with which it is degenerate, w1Il swear the peak out, perhaps to the point of not being observable.

In pure $S m$, the spectrun 5-10eV below $c_{f}$ cones from five $4 f$ electrons whereas there are three electrons, two of them d-11ke, contributing to the valence $(0-3 e V)$ spectrum. In the compound, there are again five $4 f$ electrons but there are now $48 \mathrm{Sm}$ and co valence electrons; therefore the $4 f$ spactra is expected to be less pronounced than in Sm metal. Depending on what one assunes is the background under the 5-10E region of the compound's spectrui, the "4f" Intensity is consistent (or perhape too 10w) with respect to the drop in $4 \mathrm{f}$ count relative to the band electrons. The important feature is that the structure of the 5-10eV reston is signiffeantiy different from that of pure sm. The structure and relative intenafties of the spectra of pure $S a$ and the other rare-earth metals have been shown 8,8 to be associated with the various $4 f^{-1}$ final multiplet states 9 accesfble by excitations of a 4 felectron out of the hund's rule $4 \mathrm{fn}^{\mathrm{n}}$ ground state. The $4 \mathrm{f}$ siell of $\mathrm{Sm}$ in Sicos any not be in such a ground state; the argnetic moment attributed ${ }^{\circ}$ to the shell is consistent with such a situation. The PrCos results ohed little light on this alter. The situation concerning the $4 \mathrm{f}$ shell 1 s best tested by obtaining spectra of other $\mathrm{RCO}_{5}$ compounds and it is our intent to do so. 

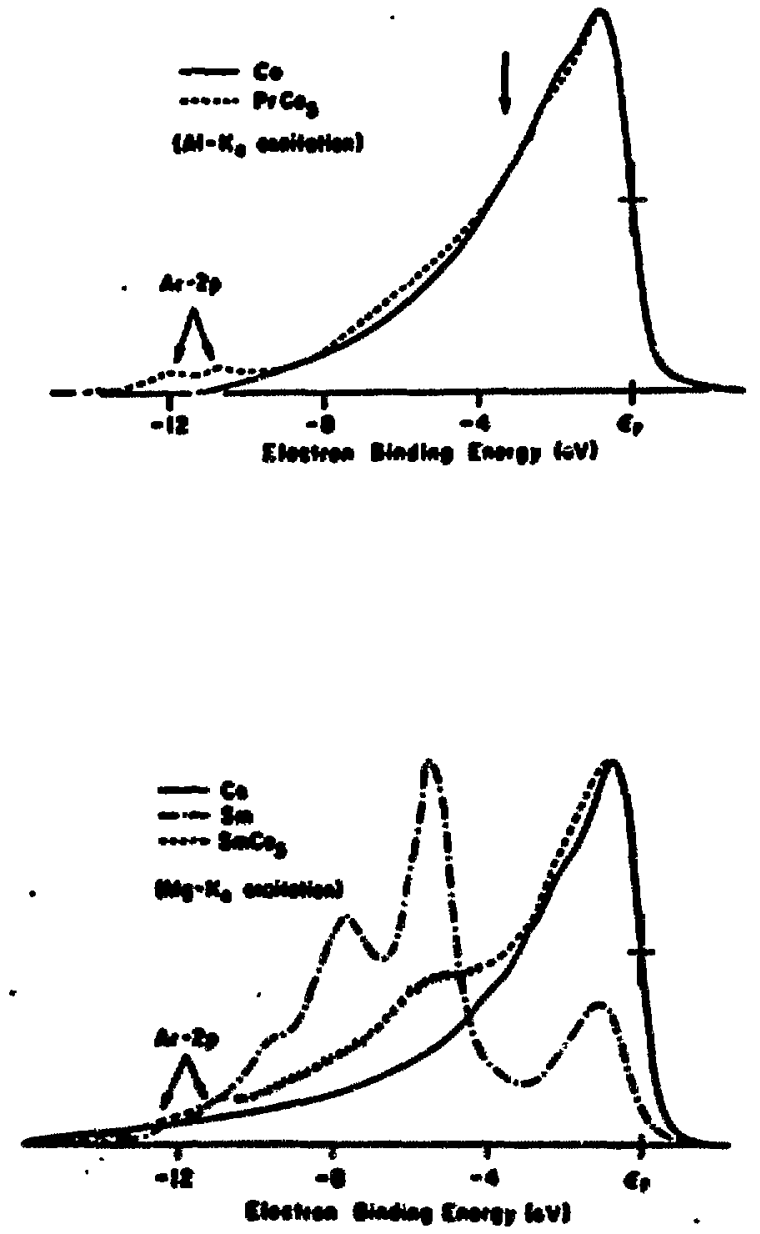

ACavinLDGALETS

I3. 2. Valence band region. Arrow lowstes position of $4 f$ peak in specterin of pure praseodyniun as deternined by Baer and Dusch. 6

F15. 3. Valence band region. The error bar: on the $\mathrm{SuCO}_{5}$ spectrue repreeent the 10cations of the data points and the alze of the randon countins exror in the crit1eal reston of the opectru discused in the text.

The authors are indebted to D. P. Fickle for making the compounde, to C. H. Brady for the metallographic studles, and to C. J. Bechtoldt for the $x$-ray diffraction analyses. The authors especially wish to thank L. H. Bennete for many st faulating discusstons. We also appreciate comunications from F. J. Arlinghave, C. $X$. Jikgensen, and $Y$. Baer. 


\section{REFFRENCES}

1. E. A. Nesbitt and J. H. Wernick, Rare Earth Permanent Magnets (Acaden1c Press, New York, 1973).

2. A. E. Ray and H. M1llott, IEEE Trans., Mag-7, 432 (1971).

3. R. Lema1re, Cobsit 32, 132 (1966).

4. See for Instance R. E. Natson, J. Hudid, and H. L. Perlman, Phys. Rev. B4, 4139 (1971).

5. D. E. Eastmun, Phys. Rev. Bi:. 1 (1970).

6. Y. Baer and G. Busch, Phys. Rev. Letters 31, 35 (1973), and to be published.

7. The band calculation of F. J. Arlinghaus (private cotanication) wy shed 11ght on this.

8. Y. Cox, Y. Baer, and C. X. J\&rgensen, cubultted to Chew. Phys. Letters, and references therein.

9. These final atates are probably screened, see J. E. Herbst, D. N. Lowy, and R. E. Watson, Phys. Rev. B6, 1913 (1972); this 1. Inportant to the position of the $4 f$ opectrum but not tis its etructure.

10. See F18. 3.2 of Ref. 1. 